\title{
High Performance Silicon Flat Optics at Visible Wavelengths
}

\author{
Arturo Burguete-Lopez ${ }^{*, 1}$, Maksim Makarenko*,1, Fedor Getman*,1, Andrea \\ Fratalocchi $^{\dagger 1}$ \\ ${ }^{1}$ PRIMALIGHT, Faculty of Electrical Engineering; Applied Mathematics and Computational Science, King \\ Abdullah University of Science and Technology, Thuwal 23955-6900, Saudi Arabia \\ ${ }^{*}$ These authors contributed equally to this work. \\ $\dagger$ Contact author: andrea.fratalocchi@kaust.edu.sa
}

\begin{abstract}
We present a platform for the design of high efficiency flat optics. Experimentally, we show common components such as polarizers, dichroics, and color filters with over $99 \%$ efficiency in the visible in 50nm of silicon. (C) 2020 The Author(s)
\end{abstract}

\section{Main Text}

Integrating conventional optics into compact nanostructured surfaces is the goal of flat optics. Despite the enormous progress of this technology, there are still critical challenges for real world applications due to a limited operational efficiency in the visible, on average lower than $60 \%$ for silicon devices, which originates by absorption losses in wavelength thick $(\approx 500 \mathrm{~nm})$ structures [1-4].

Another set of challenges lie in the design process. Historically, the search for new devices has followed what is known as the direct design approach, where the structures are limited by the intuition of the designer. Inverse design, whereby a computer designs the device given the desired optical response, has been investigated as an alternative method. Current approaches however, have been largely focused in the near infrared region [5-7], where absorption is not a concern, and haven't overcome the aforementioned problems in the visible.

In this work, we showcase an inverse design flat optics platform that employs concepts from evolutionary algorithms and deep learning with convolutional neural networks $(\mathrm{CNN})$. The platform leverages the fact that the response of a nanostructured device can be separated into resonance and propagation effects [8]. By suitably engineering the resonances of the system, our platform is able to find designs that accurately produce the desired input optical responses with ultra-thin structures, overcoming the challenge of absorption in the visible and resulting in very high efficiency devices.

To experimentally validate this approach we design, fabricate and experimentally characterize seven different structures. The manufacturing process is the same for all devices and consists of patterning a high refractive index semiconductor, deposited via PECVD onto a glass substrate, with electron beam lithography and a subsequent dry etching process with a chromium mask. We report polarizer beam splitters at $533 \mathrm{~nm}, 600 \mathrm{~nm}, 750 \mathrm{~nm}$ and $900 \mathrm{~nm}$ with experimental efficiencies as high as $99.2 \%$ and $130 \mathrm{~nm}$ bandwidth in the visible, dichroic mirrors with $90 \%$ experimental transmission/reflection efficiency and polarization dependent color filters that serve as the basis of a metasurface display technology.

\section{References}

1. B. Wang, F. Dong, Q.-T. Li, D. Yang, C. Sun, J. Chen, Z. Song, L. Xu, W. Chu, Y.-F. Xiao, Q. Gong, and Y. Li, "Visible-frequency dielectric metasurfaces for multiwavelength achromatic and highly dispersive holograms," Nano Lett. 16, 5235-5240.

2. J. Li, C. Liu, T. Wu, Y. Liu, Y. Wang, Z. Yu, H. Ye, and L. Yu, "Efficient polarization beam splitter based on all-dielectric metasurface in visible region," Nanoscale Res. Lett. 14, 34.

3. Z. Zhou, J. Li, R. Su, B. Yao, H. Fang, K. Li, L. Zhou, J. Liu, D. Stellinga, C. P. Reardon, T. F. Krauss, and $\mathrm{X}$. Wang, "Efficient silicon metasurfaces for visible light," ACS Photonics 4.

4. J. Cheng, S. Jafar-Zanjani, and H. Mosallaei, "All-dielectric ultrathin conformal metasurfaces: Lensing and cloaking applications at $532 \mathrm{~nm}$ wavelength," Sci. Reports 6, 38440.

5. B. Shen, P. Wang, R. Polson, and R. Menon, "Ultra-high-efficiency metamaterial polarizer," Optica 1, 356360.

6. D. Sell, J. Yang, S. Doshay, and J. A. Fan, "Periodic Dielectric Metasurfaces with High-Efficiency, Multiwavelength Functionalities," Adv. Opt. Mater. 5, 1700645. 

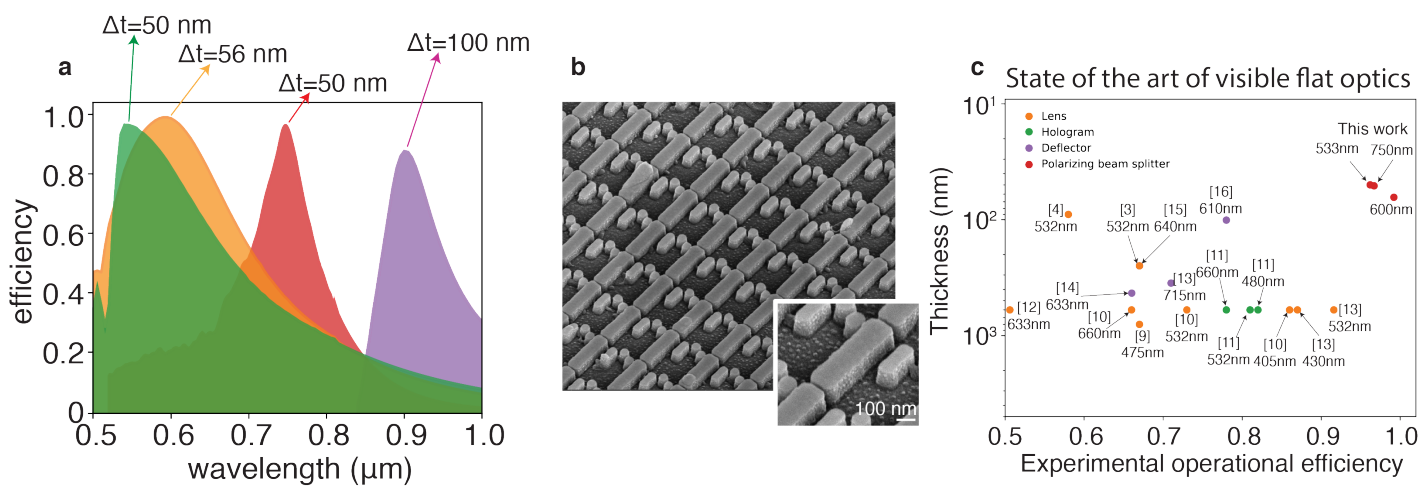

Fig. 1. (a) Experimental polarization efficiency for four polarizing beam splitters designed at common laser lines. (b) SEM micrograph showing the structure of the 900nm polarizing beam splitter. (c) Figure of merit comparing operational efficiency and thickness for the state of the art in flat optics.

7. D. Sell, J. Yang, S. Doshay, R. Yang, and J. A. Fan, "Large-Angle, Multifunctional Metagratings Based on Freeform Multimode Geometries," Nano Lett. 17, 3752-3757.

8. M. Makarenko, A. Burguete-Lopez, F. Getman, and A. Fratalocchi, "Generalized Maxwell projections for multi-mode network Photonics," Sci. Reports 10, 9038.

9. S. Wang, P. C. Wu, V.-C. Su, Y.-C. Lai, M.-K. Chen, H. Y. Kuo, B. H. Chen, Y. H. Chen, T.-T. Huang, J.-H. Wang, R.-M. Lin, C.-H. Kuan, T. Li, Z. Wang, S. Zhu, and D. P. Tsai, “A broadband achromatic metalens in the visible," Nat. Nanotechnol. 13, 227-232.

10. M. Khorasaninejad, W. T. Chen, R. C. Devlin, J. Oh, A. Y. Zhu, and F. Capasso, "Metalenses at visible wavelengths: Diffraction-limited focusing and subwavelength resolution imaging," Science 352, 1190-1194.

11. R. C. Devlin, M. Khorasaninejad, W. T. Chen, J. Oh, and F. Capasso, "Broadband high-efficiency dielectric metasurfaces for the visible spectrum,” Proc. Natl. Acad. Sci. 113, 10473-10478.

12. R. A. Aoni, M. Rahmani, L. Xu, K. Zangeneh Kamali, A. Komar, J. Yan, D. Neshev, and A. E. Miroshnichenko, "High-efficiency visible light manipulation using dielectric metasurfaces," Sci. Reports 9, 6510.

13. B. H. Chen, P. C. Wu, V.-C. Su, Y.-C. Lai, C. H. Chu, I. C. Lee, J.-W. Chen, Y. H. Chen, Y.-C. Lan, C.-H. Kuan, and D. P. Tsai, "GaN Metalens for Pixel-Level Full-Color Routing at Visible Light," Nano Lett. 17, 6345-6352.

14. A. J. Ollanik, J. A. Smith, M. J. Belue, and M. D. Escarra, "High-efficiency all-dielectric huygens metasurfaces from the ultraviolet to the infrared," ACS Photonics 5, 1351-1358.

15. T. Phan, D. Sell, E. W. Wang, S. Doshay, K. Edee, J. Yang, and J. A. Fan, "High-efficiency, large-area, topology-optimized metasurfaces," Light. Sci. \& Appl. 8, 1-9.

16. D. Sell, J. Yang, S. Doshay, K. Zhang, and J. A. Fan, "Visible Light Metasurfaces Based on Single-Crystal Silicon," ACS Photonics 3, 1919-1925.

Acknowledgments This research received funding from KAUST (Award OSR-2016-CRG5-2995).

Competing Interests A provisional patent application (US Patent Application No. 62/844,416) has been filed on the basis of the results of this work. 